University of Nebraska - Lincoln

DigitalCommons@University of Nebraska - Lincoln

Faculty Publications, Department of Physics and Astronomy

Research Papers in Physics and Astronomy

6-21-2004

\title{
Simulations of ferroelectric polymer film polarization: The role of dipole interactions
}

\author{
Chun-gang Duan \\ University of Nebraska-Lincoln, cgduan@clpm.ecnu.edu.cn \\ Wai-Ning Mei \\ University of Nebraska at Omaha, physmei@unomaha.edu \\ Wei-Guo Yin \\ Zhejiang University \\ Jianjun Liu \\ University of Nebraska-Omaha, jianjunliu@mail.unomaha.edu \\ J. R. Hardy \\ University of Nebraska-Omaha
}

See next page for additional authors

Follow this and additional works at: https://digitalcommons.unl.edu/physicsfacpub

Part of the Physics Commons

Duan, Chun-gang; Mei, Wai-Ning; Yin, Wei-Guo; Liu, Jianjun; Hardy, J. R.; Ducharme, Stephen; and Dowben, Peter A., "Simulations of ferroelectric polymer film polarization: The role of dipole interactions" (2004). Faculty Publications, Department of Physics and Astronomy. 13.

https://digitalcommons.unl.edu/physicsfacpub/13

This Article is brought to you for free and open access by the Research Papers in Physics and Astronomy at DigitalCommons@University of Nebraska - Lincoln. It has been accepted for inclusion in Faculty Publications, Department of Physics and Astronomy by an authorized administrator of DigitalCommons@University of Nebraska - Lincoln. 


\section{Authors}

Chun-gang Duan, Wai-Ning Mei, Wei-Guo Yin, Jianjun Liu, J. R. Hardy, Stephen Ducharme, and Peter A. Dowben 


\title{
Simulations of ferroelectric polymer film polarization: The role of dipole interactions
}

\author{
Chun-gang Duan, ${ }^{1, *}$ W. N. Mei, ${ }^{1}$ Wei-Guo Yin, ${ }^{1}$ Jianjun Liu, ${ }^{1,2}$ J. R. Hardy, ${ }^{1,2}$ Stephen Ducharme, ${ }^{2}$ and P. A. Dowben ${ }^{2}$ \\ ${ }^{1}$ Department of Physics, University of Nebraska at Omaha, Omaha, Nebraska 68182-0266, USA \\ ${ }^{2}$ Department of Physics and Center for Materials Research and Analysis, University of Nebraska at Lincoln, Lincoln, \\ Nebraska 68588, USA \\ (Received 11 December 2003; published 21 June 2004)
}

\begin{abstract}
We present a systematic study of the dipole alignment in the polyvinylidene fluoride (PVDF) films using first-principles total energy calculations. The ground state of a single layer film is a state with all the dipoles lying parallel to the film plane. This can also be explained by a dipole-dipole interaction model. The induced mirror charges on conducting substrates or substrates with a non-negligible dielectric response play an important role in aligning the polarization perpendicular to the film. From fitting the $a b$ initio calculations, we obtain an effective monomer dipole moment of $4.7 \times 10^{-30} \mathrm{C} \mathrm{m}$. This corresponds to a spontaneous polarization of $0.087 \mathrm{C} / \mathrm{m}^{2}$, which agrees with other theoretical and experimental values. Simulation reveals a more complex behavior for molecular bilayer. We studied three molecular multilayer structures to compare the total energy and model calculations. Close examination of these results provides a better understanding of PVDF film growth and dipole orientation on different substrates.
\end{abstract}

DOI: 10.1103/PhysRevB.69.235106

PACS number(s): 77.84.Jd, 71.20.-b, 68.55.-a

\section{INTRODUCTION}

The pursuit of nonvolatile random-access memory devices and nanoscale electronic devices has provided impetus to the study of ferroelectric thin films. ${ }^{1,2}$ Ferroelectricity is difficult, generally, to maintain when the size of the ferroelectric material is greatly reduced. ${ }^{3}$ Furthermore, the growth of thin insulating films depends strongly on the substrate., 4 This makes the fabrication of the ultrathin ferroelectric film very difficult.

Ferroelectric polymers [polyvinylidene fluoride (PVDF)] provide an alternative way to fabricate high-quality ultrathin crystalline ferroelectric films. ${ }^{6}$ For most device applications, the polar direction has to be perpendicular to the film. The PVDF polymer films can be grown on conductors like graphite, and the dipoles are aligned generally perpendicular to the surface, ${ }^{7}$ but on some substrates like $\mathrm{KCl}$, for vinylidene fluoride (VDF) oligomer thin films, the electric dipole moments are parallel to the substrate. ${ }^{8}$ Precise nanoscale polarization manipulation and conductance switching have been carried out on these films. ${ }^{9}$ Molecular reorientations and lattice shift are observed by flipping the polarity of the scanning tunneling microscope (STM), clearly demonstrated local polarization reversal. The mechanism of the molecular reorientations has not been conclusively established. ${ }^{9}$

To better understand the origins of preferential dipole orientation in the ferroelectric polymers, we carried out theoretical simulations. Based on ab initio total energy calculations on PVDF slabs, we introduced a dipole model that simplifies the chain-chain interaction in the PVDF film.

\section{CALCULATIONAL APPROACH AND MODEL STRUCTURE}

The first-principles band structure approach applied in this work is the full-potential linear-augmented-plane-wave (FLAPW) method $^{10}$ with the exchange-correlation potential treated in the generalized gradient approximation ${ }^{11}$ (GGA) of the density functional theory. We have used the same approach to study the energy band structure, band symmetries, and optical properties of the $\beta$ phase of PVDF. ${ }^{12,13}$ The calculated results agree well with photoemission experiments. ${ }^{7}$

The electronic properties of PVDF films are simulated by a periodically repeated slab model based on the crystal structure of the ferroelectric phase ( $\beta$ phase) of PVDF, which is an all-trans planar zigzag structure with all chain dipoles oriented along the twofold axis. ${ }^{12}$ To simplify the calculation, we ignore the $7^{\circ}$ deflections in the PVDF chain; thus the lattice constant a (the chain direction) of the supercell is half of the $\mathbf{c}$ of crystal. ${ }^{12}$ The lattice constant $\mathbf{c}$ of the supercell is set to be $24.6 \AA$, 5 times that of the $\mathbf{b}$ (polar axis) of the crystal $\beta$ phase, which ensures the layers are well isolated. The $\mathbf{b}$ of the supercell has the value of the lattice constant a of the crystal PVDF.

As in previous calculations, ${ }^{12,13}$ the muffin-tin (MT) radii of $\mathrm{C}, \mathrm{H}$, and $\mathrm{F}$ atoms are chosen as 1.1, 0.7, and 1.4 a.u., respectively. The energy cutoff for plane wave expansions in the interstitial region is above $18 \mathrm{Ry}$. The upper limit of the angular momentum, $l_{\max }=10$, is adopted in the sphericalharmonic expansion of the Kohn-Sham functions inside the atom spheres. Eight $k$ points in the irreducible part of the Brillouin zone are used in the self-consistent calculation. The convergence obtained is up to $0.2 \mathrm{meV}$ of the total energy.

\section{SIMULATION ON MONOLAYER PVDF SLAB}

We first carried out the total energy calculation on a monolayer slab. Noticing that the dipoles only rotate around the chain axis, ${ }^{9}$ we then studied the relationship between the total energy (per unit cell) and the dipole rotation angle with respect to the surface normal. The chain-chain spacing is kept as constant during the rotation. The results are shown in Fig. 1(a).

In the ground state of the single PVDF layer, we find that all the dipoles lie flat and parallel to the surface plane. This 

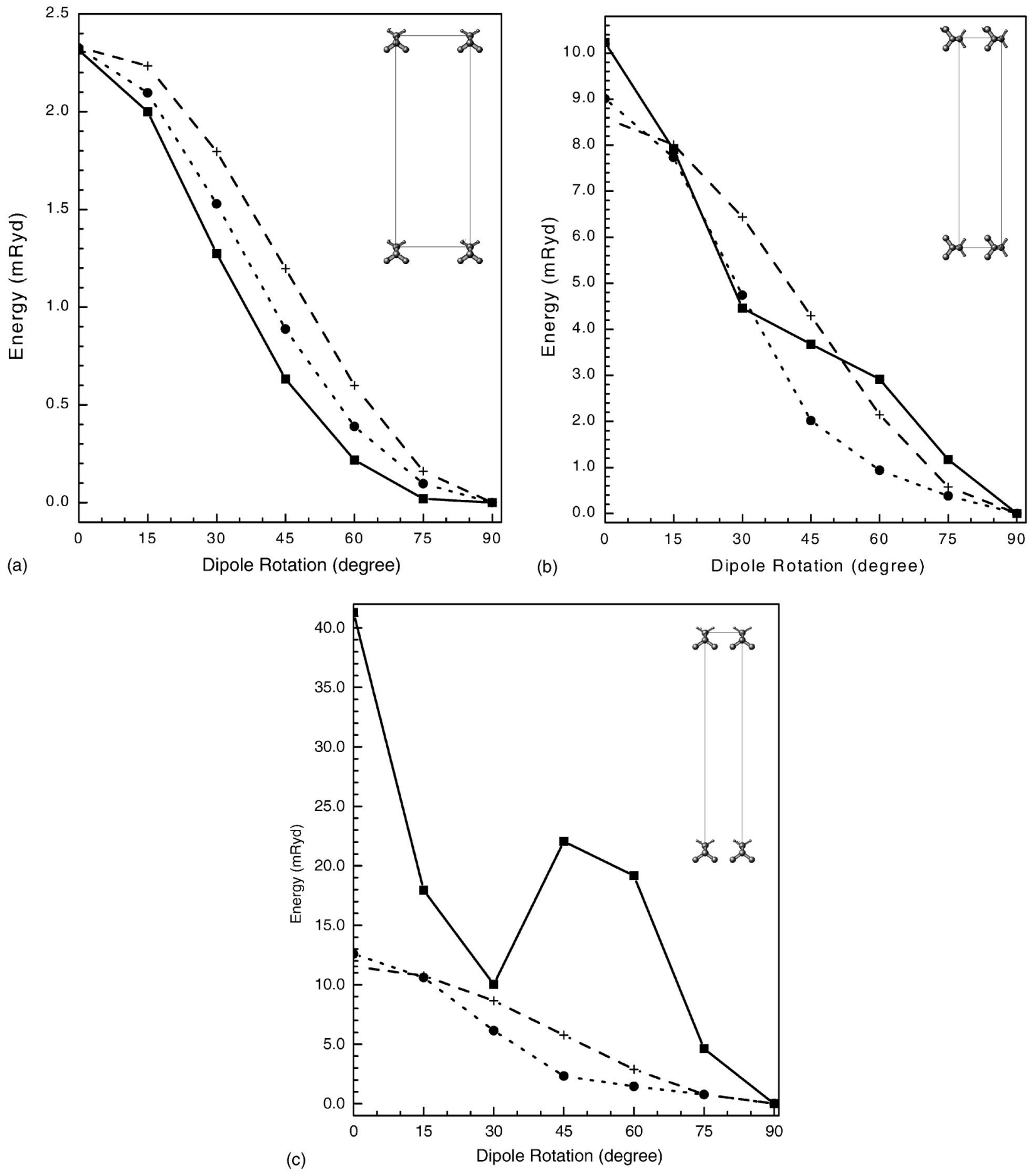

FIG. 1. Calculated energy of monolayer PVDF film as a function of the dipole rotation around the PVDF chain (where $0^{\circ}$ is along the surface normal) using the FLAPW (solid), dipole (dashed), and point charge (dotted) calculational models. The chain-chain spacing are (a) $8.58 \AA$, (b) $4.91 \AA$, (c) $4.3 \AA$. The energy bases are $-554.2160,-554.3141$, and -554.3388 Ry, respectively. Insets are the demonstration plots of the supercells.

conclusion is supported by experiments of VDF oligomer thin film growth, ${ }^{8}$ but does not agree with the experimental observations of Ref. 7. Hence the choice of substrate appears to be a major consideration. The substrates used in Ref. 7 were generally conductors, e.g., graphite or highly doped silicon, while those for VDF oligomer are alkali halide such as $\mathrm{KCl}$, which are insulators. Thus, it is easy to see that the final dipole orientations of the monolayer film depend strongly on the interaction between the dipoles and substrate. As we know, the PVDF polymer dipoles are large, and the dipoles would certainly induce mirror charges on a conducting substrate. These induced mirror charges exert an extrinsic vertical electric field on the adsorbed slab, causing the dipoles to stand on the surface plane. Therefore, in this sense, 
the Langmuir-Blodgett monolayer PVDF film is actually an extrinsic ferroelectric. This is an example of the way in which a conducting layer provides the surface charge necessary to cancel out the depolarizing field which otherwise suppresses ferroelectricity. ${ }^{14} \mathrm{On}$ an insulating substrate such as $\mathrm{KCl}$, there are little or no mirror charges, and the film grows with all the dipoles lying flat in the surface plane. This does not imply that there is no interaction between an insulating substrate and the PVDF dipole orientation and placement. In contrast, as observed in VDF oligomer thin films on a $\mathrm{KCl}$ substrate, ${ }^{8}$ the $\mathrm{F}$ atoms of the PVDF monomer are located on $\mathrm{K}^{+}$and $\mathrm{H}$ atoms on $\mathrm{Cl}^{-}$along the $\mathrm{KCl}[110]$ directions, clearly indicating a strong electrostatic action between the sample and substrate. And this kind of interaction tends to strengthen the tendency to align the dipoles parallel to the substrate.

Describing the PVDF chain as an infinite dipole array, we then modeled the interaction between PVDF chains as the sum of the dipole-dipole interaction across the chains. It is well known that the interaction energy between two dipoles is given by ${ }^{15}$

$$
W_{12}=\frac{\mathbf{p}_{1} \cdot \mathbf{p}_{2}-3\left(\mathbf{n} \cdot \mathbf{p}_{1}\right)\left(\mathbf{n} \cdot \mathbf{p}_{2}\right)}{\left|\mathbf{r}_{1}-\mathbf{r}_{2}\right|^{3}},
$$

where $\mathbf{p}_{1}$ and $\mathbf{p}_{2}$ are dipole moments, and $\mathbf{n}$ is a unit vector in the direction $\left(\mathbf{r}_{1}-\mathbf{r}_{2}\right)$. In order to compare with the FLAPW total energy calculations of the slab, the quasi-threedimensional (3D) effect should be taken into account, i.e., we should also sum along the $\mathbf{z}$ direction. Convergence of the lattice sum over this PVDF slab structure is good. We found that there is no more than $1 \%$ difference between the results obtained by a cutoff distance of 1000 and $5000 \AA$. After fitting the energy base and dipole moment (note that we use this dipole moment throughout the entire paper), our dipolar simulation results agree well with the total energy calculations [see also Fig. 1(a)].

We would like to point out that the so obtained dipole moment can be directly compared with experimental observations. The fitted monomer dipole moment is 4.7 $\times 10^{-30} \mathrm{C} \mathrm{m}$, which corresponds to a spontaneous polarization of $0.087 \mathrm{C} / \mathrm{m}^{2}$ for the crystal $\beta$ phase. Experimental values of the remenant polarization are varied from 0.05 to $0.065 \mathrm{C} / \mathrm{m}^{2}$, depending on the purity (generally $50 \%$ ) and crystallinity of the samples used in experiments. Recent experiments on a highly crystalline two-dimensional PVDF film obtained a value of $0.1 \mathrm{C} / \mathrm{m}^{2}{ }^{6}$ Our calculation agrees quite well with experimental observations. In addition, our effective dipole moment also agrees well with the value $4.62 \times 10^{-30} \mathrm{C} \mathrm{m}$, obtained in a sophisticated way that involves calculation of the Lorentz factor of an orthorhombic crystal. ${ }^{16,17}$

To examine whether the small difference between these two results was caused by the multipole effect, we also used the point charge model to calculate the energy variations. By fitting the total energy curve, we obtained the charges associated with each atom as $Q_{\mathrm{CH}}=-0.52$ (electron units), $Q_{\mathrm{H}}$ $=0.17, Q_{\mathrm{CF}}=0.68, Q_{\mathrm{F}}=-0.25$, where $\mathrm{CH}$ and $\mathrm{CF}$ indicate the carbon bonded to $\mathrm{H}$ and $\mathrm{F}$, respectively. We noticed that these values differ only slightly from those applied to the force field calculation: $-0.54,0.18,0.70$, and -0.26 , by Karasawa and Goddard. ${ }^{18}$ The cutoff distance $1000 \AA$ appears to be good enough to obtain the accuracy needed in current calculation, indicating that the complicated acceleration method for lattice sums such as the Ewald technique is not necessary. As can be seen from Fig. 1(a), considering the multipole effect does lead to a better agreement with the total energy calculation, but it still could not account for the rest of the discrepancies, which might result from the exact charge density distribution.

As observed in the VDF oligomer on $\mathrm{KCl}^{8}{ }^{8}$ the lattice spacing on the same layer is actually the crystal lattice constant b. Thus we also repeated the same procedure mentioned above on a supercell with its $\mathbf{b}$ changed to the value of crystal lattice constant b $(4.91 \AA)$. The results are shown in Fig. 1(b). As we can see from the figure, the same conclusion as in previous case can be drawn, just the discrepancies between the total energy calculation and dipole model calculations became larger. This disagreement could not be caused by the multipole effect (as shown in point charge model calculation); thus a more plausible explanation is the dipole moment change, or, more precisely, the charge redistribution caused by the distance shortening and chain rotations.

Similarly, in Fig. 1(c) we depict our calculated result for a supercell with $4.3 \AA$ in-plane spacing, as observed experimentally on the surface crystal structure. ${ }^{19}$ This time, because of the reason mentioned above, the agreement between these three approaches are not as good as those in the previous examples, indicating that assuming the dipole and point charges to be constants during the rotation is unsatisfactory when the chain-chain spacing becomes small. Nevertheless, all three calculations provide similar trends of the energy change with rotation.

\section{SIMULATION ON BILAYER PVDF SLAB}

To study the interlayer coupling effect, we carried out calculations on a two-layer PVDF slab. As expected, the situation becomes much more complicated. We chose three typical structures to elucidate our analysis: in the first case [Fig. 2(a)], the PVDF chains of the top layer were located $4.91 \AA$ above those in the ground (interface) layer, where the dipole orientations are pinned normal to the film surface. The dipoles of the top layer were then allowed to rotate from 0 to $180^{\circ}$. As we can see from the figure, the dipole and point charge model predict the energy maximum at $180^{\circ}$, which agree with the FLAPW calculations. But both of their minima locate at $60^{\circ}$, mismatched with the FLAPW value, $0^{\circ}$.

To simulate the crystal $\beta$ phase, in the second case [Fig. 2(b)], the chains in the top layer were horizontally shifted by $4.29 \AA$, half of the lattice constant $\mathbf{b}$ of the supercell, and the vertical spacing drops to $2.46 \AA$. This time we see that all three calculations give the energy minimum closely (around $120^{\circ}$ ). But the energies given by model calculations at $0^{\circ}$ and $180^{\circ}$ are quite different from the FLAPW calculations. We 

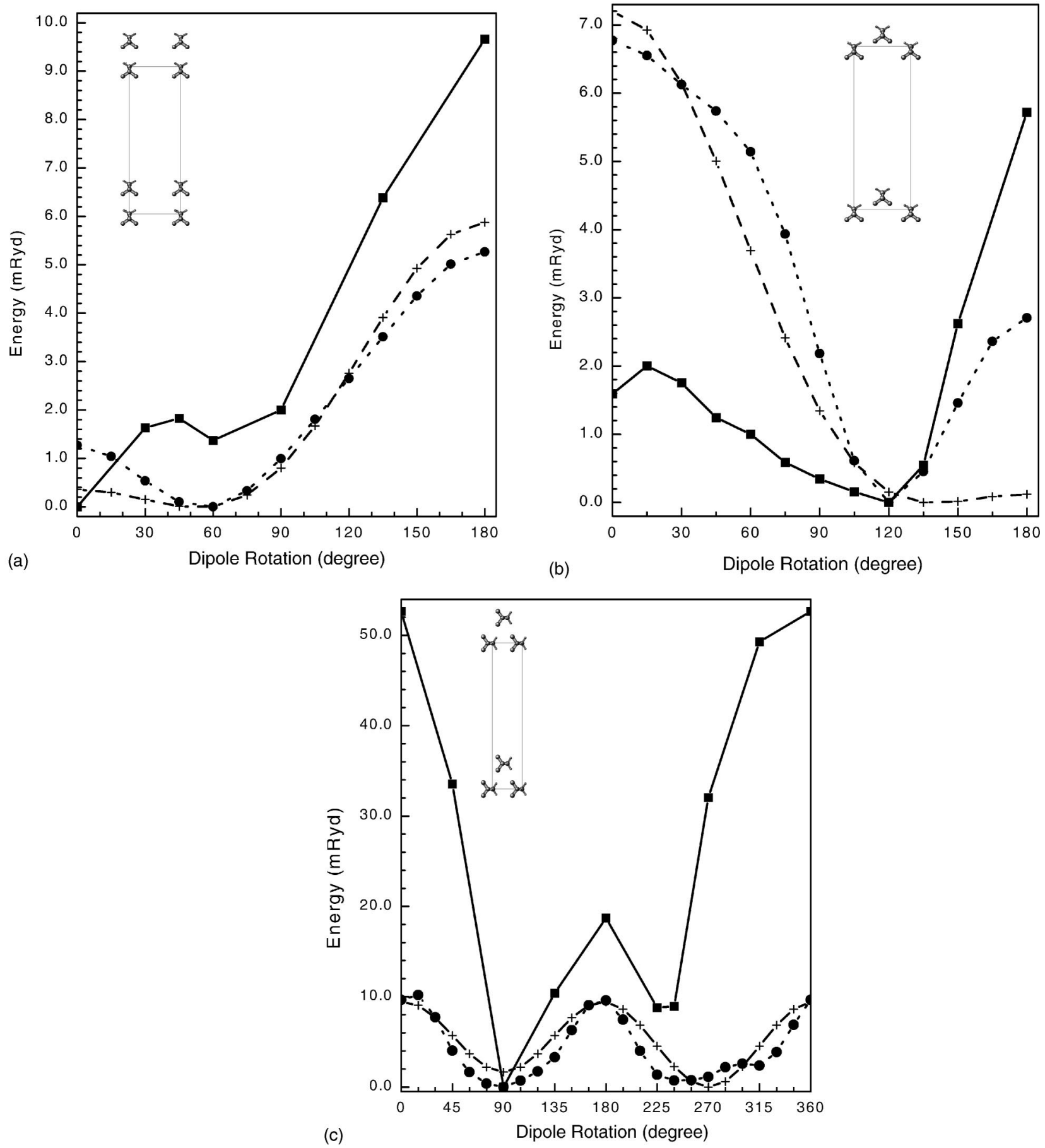

FIG. 2. Same as Fig. 1, except this is for a two-layer PVDF film and the dipoles of the ground layer are pinned at (a) $0^{\circ}$, layer spacing $4.91 \AA$, no horizontal shift between two layers; (b) $0^{\circ}$, layer spacing $2.46 \AA$, horizontal shift: $4.29 \AA$; (c) $90^{\circ}$, layer spacing $4.29 \AA$, horizontal shift $2.46 \AA$. The energy bases are, $-1108.4322,-1108.4307$, and -1108.6387 Ry, respectively.

also noticed that in this case the curves given by the point dipole and point charge calculations have different shapes, indicating the significant finite size dipole effect. This is also supported by the calculation of Purvis and Taylor. ${ }^{17}$

In the third case [Fig. 2(c)], we tried to simulate the situation of the crystal growth on insulators, where the dipoles lie parallel to the surface. ${ }^{8}$ The supercell lattice constant $\mathbf{b}$ is chosen to be crystal $\mathbf{b}$ with the dipoles of the ground layer pinned at $90^{\circ}$, and the top layer was located $4.29 \AA$ above the ground layer and was shifted horizontally by $2.46 \AA$. The FLAPW calculation predicted that to achieve the ground state of this two-layer structure, the dipoles of the top layer should also be parallel to the surface and ground layer dipoles, which agrees with experimental observations and model calculations. Actually both model calculations predict a double-well structure for the energy variation of the dipole 
rotation. The point charge model calculations gives two energy minima at $90^{\circ}$ and $240^{\circ}$, same as those of the FLAPW observations. In dipole model calculation, those two minima are $90^{\circ}$ (parallel) and $270^{\circ}$ (antiparallel). It gives a little higher energy at $90^{\circ}$ than that at $270^{\circ}$. But if we ignore the slab model effect, the dipole model would also predict the lowest energy at $90^{\circ}$. Furthermore, the dipole model calculation also demonstrated that this minimum is actually a global minimum when we shift the second layer by keeping the interlayer chain-chain spacing to no more than $4.94 \AA$. This provides some insight into how the VDF oligomer thin film grows layer by layer on an insulating substrate.

\section{DISCUSSIONS AND CONCLUSIONS}

From the above observations, we found that our theoretical calculations can well describe the crystal growth on the more insulating substrate. The energy minimum in this case [see Fig. 2(c)] given by the FLAPW calculation is $-1108.6387 \mathrm{Ry}$, which is lower than those of the other two cases ( -1108.4322 and $-1108.4307 \mathrm{Ry})$. This indicates that the ground state of a substrate-free bilayer slab is a state with all the dipoles of two layers lying parallel to the film surface. This is a nontrivial conclusion, since the interlayer interaction would become more and more important when the layer thickness increases. Thus, similar to the monolayer case, if the film grows on a conductive substrate, the substrate effect has to be considered. For example, since the energy difference given by the FLAPW calculation at 0 and $120^{\circ}$ is only $1.5 \mathrm{mRy}$, the mirror charge would easily align the dipoles of the top layer to be normal to the dipole surface, as observed experimentally. ${ }^{6}$

To explain the discrepancies between the total energy and model calculations, we believe the charge redistribution during the rotation is essential, i.e., keeping the dipole moment fixed in the study is only an approximation to the real situation. As can be seen from Fig. 2(c), model calculations give same values at 0 and $180^{\circ}$, but the FLAPW calculation gives a much lower energy at $180^{\circ}$ than at $0^{\circ}$. Considering the close proximity between the top and ground layer, the charge distribution on each atom at these two states will not remain identical. Remembering that the interaction energy is proportional to the square of dipole moment, this would result in significant discrepancy between the total energy and model calculations.

Overall, we have found that the dipole model could well present the PVDF chain-chain interaction. It agrees with the total energy calculations especially well for the monolayer case, when the PVDF chains are separated far enough (e.g., $>5.0 \AA$ ). As we have pointed out in our previous paper, ${ }^{12}$ the energy dispersion of the valence band is very small along the direction perpendicular to the PVDF chain, which indicates weak chain-chain interactions. Here the successful application of the dipole model further demonstrates that the PVDF chain-chain interaction is mainly electrostatic. Similar situations have been reported for the complex perovskite alloys. ${ }^{20}$ It is possible that there are a number of examples where the sophisticated energy band structure calculations can be verified by a simple dipole model calculation.
For the freestanding monolayer case, the PVDF dipoles tend to lie on the film surface. Dipole orientations become complicated when the PVDF film is placed on conducting substrates. First-principles calculations involving the interaction between conducting substrates and the PVDF film are currently too difficult to be undertaken. We can, however, estimate the effect of the substrate by comparing the isolated slab model calculation and experimental observations. Based on the above analysis we conclude that there must exist an electric field perpendicular to the film that aligns the dipoles along the surface normal. If the electric field at the interface with the substrate is not very strong, dipoles in the polymer thin film might be canted with respect to the film normal, as a result of the interplay between two competitive mechanisms influencing dipole orientations.

In considering recent STM observations, ${ }^{9}$ since we found that the behavior of the PVDF chains and dipole orientation could be described reasonably well as a dipole-dipole interaction and the estimated effective dipole moment agrees well with experiments and other calculations, the picture became much clearer. We believe that the lattice shift induced by flipping the STM tip bias voltage was caused by the flipping of the local dipole underneath the STM tip when the voltage was reversed. The reversal of such a dipole would involve a local structural change in which nearby dipoles are forced to rotate $90^{\circ}$, and render a lattice shift in the STM image. Since it requires a finite electric field to overcome the energy barrier and flip the dipole, no lattice shift was observed when the tip bias is too low $(<0.1 \mathrm{~V})$. On the other hand, if the tip bias is too large, the polarization reversal is no longer a local behavior, i.e., the entire top layer (at least a quite large range) is polarized; hence the lattice shift will not be observed either.

In conclusion, our $a b$ initio total energy calculations on the PVDF slab shows that the ground state of an isolated PVDF film is a state with all the dipoles lying flat and parallel to the surface plane. However, this is not the case for PVDF ultrathin films on the conductive substrate, because image dipoles tend to force the PVDF dipole to orientate along the surface normal. The interaction between the substrate and the PVDF film plays a crucial role in orientating the PVDF dipole direction. Introducing the dipole-dipole interactions to explain the results of the total energy calculation appears to be effective. We have found that the dipole model describes satisfactorily the behavior of the PVDF chain-chain interactions. Our studies provide insight into the mechanisms affecting the growth of PVDF ultrathin films and dipole orientations on different substrates and the behavior of PVDF films under the STM tip.

\section{ACKNOWLEDGMENTS}

The authors thank Professor L. L. Boyer for fruitful discussion. This work was supported by the Office of Naval Research, Nebraska Research Initiative, and Army Research Office. 
*Email address: dcg@ cosmos.unomaha.edu

${ }^{1}$ S. Mathews, R. Ramesh, T. Venkatesan, and J. Benedetto, Science 276, 238 (1997).

${ }^{2}$ O. Auciello, J. F. Scott, and R. Ramesh, Phys. Today 51 (July), 22 (1998).

${ }^{3}$ T. Tybell, C. H. Ahn, and J.-M. Triscone, Appl. Phys. Lett. 75, 856 (1999).

${ }^{4}$ Marshall Stoneham, Nature (London) 410, 159 (2001).

${ }^{5}$ Jascha Repp, Stefan Fölsch, Gerhard Meyer, and Karl-Heinz Rieder, Phys. Rev. Lett. 86, 252 (2001).

${ }^{6}$ A. V. Bune, V. M. Fridkin, Stephen Ducharme, L. M. Blinov, S. P. Palto, A. V. Sorokin, S. G. Yudin, and A. Zlatkin, Nature (London) 391, 874 (1998).

${ }^{7}$ Jaewu Choi, S.-J. Tang, P. T. Sprunger, P. A. Dowben, V. M. Fridkin, A. V. Sorokin, S. P. Palto, N. Petukhova, and S. G. Yudin, J. Phys.: Condens. Matter 12, 4735 (2000).

${ }^{8}$ Kei Noda, Kenji Ishida, Toshihisa Horiuchi, Kazumi Matsushige, and Atsushi Kuboono, J. Appl. Phys. 86, 3688 (1999).

${ }^{9}$ Hongwei Qu, Wei Yao, T. Garcia, Jiandi Zhang, A. V. Sorokin, S. Ducharme, P. A. Dowben, and V. M. Fridkin, Appl. Phys. Lett. 82, 4322 (2003).

${ }^{10}$ P. Blaha, K. Schwarz, G. K. H. Madsen, D. Kvasnicka, and J.
Luitz, WIEN2k, An Augmented Plane Wave + Local Orbitals Program for Calculating Crystal Properties (Karlheinz Schwarz, Techn. Universität Wien, Austria, 2001).

${ }^{11}$ J. P. Perdew, K. Burke, and M. Ernzerhof, Phys. Rev. Lett. 77, 3865 (1996).

${ }^{12}$ C. G. Duan, W. N. Mei, J. R. Hardy, S. Ducharme, J. Choi, and P. A. Dowben, Europhys. Lett. 61, 81 (2003).

${ }^{13}$ C. G. Duan, W. N. Mei, W. G. Yin, J. Liu, J. R. Hardy, M. Bai, and S Ducharme, J. Phys.: Condens. Matter 15, 3805 (2003).

${ }^{14}$ J. R. Hardy and H. M. Lu, Ferroelectrics 136, 167 (1992).

${ }^{15}$ John David Jackson, Classical Electrodynamics (Wiley, New York, 1969), p. 102.

${ }^{16}$ C. K. Purvis and P. L. Taylor, Phys. Rev. B 26, 4547 (1982).

${ }^{17}$ C. K. Purvis and P. L. Taylor, J. Appl. Phys. 54, 1021 (1983).

${ }^{18}$ N. Karasawa and W. A. Goddard III, Macromolecules 25, 7268 (1992).

${ }^{19}$ Jaewu Choi, C. N. Borca, P. A. Dowben, A. Bune, M. Poulsen, Shawn Pebley, S. Adenwalla, Stephen Ducharme, Lee Robertson, V. M. Fridkin, S. P. Palto, N. Petukhova, and S. G. Yudin, Phys. Rev. B 61, 5760 (2000).

${ }^{20}$ L. Bellaiche and David Vanderbilt, Phys. Rev. Lett. 81, 1318 (1998). 